\section{Estructura factorial de la escala DREEM en estudiantes de medicina chilenos}

\author{
JAVIERA ORTEGA B. ${ }^{1, a}$, CRISTHIAN PÉREZ V. ${ }^{1, b}$, \\ LILIANA ORTIZ M. ${ }^{1, \mathrm{c}}$, EDUARDO FASCE H. ${ }^{1}$, \\ PETER MCCOLL C. ${ }^{2, \mathrm{~d}}$, GRACIELA TORRES A. ${ }^{3, \mathrm{e}}$, ANA WRIGHT ${ }^{4, \mathrm{f}}$, \\ CAROLINA MÁRQUEZ U. ${ }^{1, \mathrm{~g}}$, PAULA PARRA P. ${ }^{1, \mathrm{~h}}$
}

\section{An assessment of the Dundee ready education environment measure (DREEM) in Chilean university students}

Background: The entry to a University requires an adaptation process that not all students solve with the same kind of success. Even though students' social adaptation and emotional skills are essential, the educational environmental that they perceive has a significant influence in their academic life. Aim: To describe the changes in the perception about academic environment that medical students experience during the first three years of undergraduate career. Material and Methods: The Dundee Ready Education Environment Measure (DREEM) scale was applied to 525 first to third year medical students and an exploratory factorial analysis was made. Results: Four factors were identified: Academic Perception: academic quality that students attribute to the process in which they take part, as well as to the assessment that they do of their learning outcomes (coefficient $\alpha=0.85$ ); Academic Experience: refers to positive emotions that students experience during the career such as confidence, pleasure and energy (coefficient $\alpha=0.76$ ); Atmosphere Perception, comfort and calm that students experiment during their academic activities (coefficient $\alpha=0.79$ ); Teachers Perception: the perception that students have of teachers about their interest and disposition towards students (coefficient $\alpha=0.50$ ). Conclusions: The assessment of academic environment quality is inversely associated with the lapse that the students have spent in their undergraduate careers.

(Rev Med Chile 2015; 143: 651-657)

Key words: Education, Medical; Psychometrics; Students, medical; Undergraduate.
'Departamento de Educación Médica, Facultad de Medicina, Universidad de Concepción, Chile.

${ }^{2}$ Facultad de Medicina, Universidad Andrés Bello, sede

Viña del Mar, Chile.

${ }^{3}$ Facultad de Medicina,

Universidad San Sebastián, Chile.

${ }^{4}$ Centro de Educación Médica,

Pontificia Universidad Católica

de Chile.

assicóloga, Magíster en

Ciencias de la Educación

Mención Didáctica e Innovación

Pedagógica.

bPsicólogo, Magíster en Psicología con mención en Psicología

Educativa.

'Magíster en Educación Superior.

'Médico cirujano, Magíster

en Educación Médica para las

Ciencias de la Salud.

eEnfermera Matrona, Magíster en Educación Médica para las

Ciencias de la Salud.

fProfesora, Licenciada en

Educación.

'Bioquímico, Magíster en

Educación Médica para las Ciencias de la Salud.

hKinesióloga, Magíster en Educación Médica para las Ciencias de la Salud.

Trabajo financiado por proyecto FONDECYT No 1121002.

Recibido el 1 de diciembre de 2014, aceptado el 3 de abril de 2015.

Correspondencia a: Javiera Ortega B.

Departamento de Educación Médica, Facultad de Medicina Universidad de Concepción Barrio Universitario s/n, Concepción

Chile.

javieraortega@udec.cl

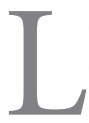

os acelerados cambios sociales y tecnológicos que ocurren a nivel mundial, han obligado a las universidades a revisar su quehacer de forma integral. Los procesos de cambios afectan a estas instituciones educativas por ser estas las responsables de la generación de conocimiento y formación de profesionales ${ }^{1}$. Esto ha provocado que las instituciones de educación superior desarrollen programas y proyectos que contribuyan a solucionar los problemas nacionales, a través de la formación de educadores, la investigación educativa y la extensión ${ }^{2}$. Se ha hecho necesario, por tanto, contar con una serie de elementos: programas de permanente formación y actualización de docentes-investigadores; la creación, renovación, crecimiento y mantenimiento de la infraestructura de bibliotecas, laboratorios y locales para la investigación, y por último, la creación de grupos multidisciplinarios y de líneas de investigación ${ }^{3}$. Por su parte, Gros ${ }^{4}$ enfatizó que 
tomando en cuenta la investigación en el ámbito de la educación, las carreras universitarias se verán enfrentadas a los procesos de cambio e innovación en la enseñanza universitaria que darán cuenta de mejoras en el proceso de enseñanza-aprendizaje.

La Educación Médica también ha realizado aportes importantes en la innovación de la enseñanza universitaria y un aspecto que ha sido considerado fundamental en el proceso de enseñanza-aprendizaje es el ambiente educativo y los factores que influyen en éste ${ }^{5,-10}$. El impacto del clima educativo ha sido reconocido por la influencia que éste ejerce en la satisfacción y éxito de los estudiantes ${ }^{11}$. Sin embargo, promover un clima positivo requiere generar cambios en una cultura organizacional que avance hacia la innovación ${ }^{12}$. Para esto no sólo se deben incluir modificaciones en la forma de implementar el currículo ${ }^{13}$, sino que también surge la necesidad de contar con un instrumento válido que permita a las carreras del área de la salud detectar aquellas variables que influyen en el ambiente educativo.

A lo largo de la investigación en educación médica se han desarrollado diversos instrumentos que permiten evaluar el clima educativo. Uno de los primeros instrumentos diseñado fue el $\mathrm{Me}$ dical School Environment Index (MSEI), creado por Hutchins ${ }^{14}$, el cual consta de 180 ítems y 18 dimensiones o sub-escalas. A partir de los resultados obtenidos por Hutchins ${ }^{14}$, se comenzaron a diseñar otros instrumentos que tienen como objetivo medir el ambiente educativo hospitalario. Uno de los más utilizados actualmente es el Dundee Ready Education Environment Measure $(\text { DREEM })^{15,16,17,18,19}$, diseñado por Genn y Harden y mejorado por Roff et $\mathrm{al}^{5}$.

Este instrumento consta de 50 ítems que, de acuerdo a los autores, se agrupan en cinco dominios o factores ${ }^{5}$ : Percepción del aprendizaje, que tiene relación con la visión que tienen los estudiantes sobre las actividades de enseñanza, asociado a cómo recibir objetivos claros por parte de sus docentes y si éstos realizan una enseñanza centrada en los estudiantes y promueven un aprendizaje activo; Percepción de los docentes, que hace referencia a la calidad de los docentes traducida en habilidades comunicacionales efectivas, si entregan retroalimentación a los estudiantes y pacientes, y el nivel de preparación de clases; Percepción Académica, se caracteriza por las estrategias de aprendizaje y habilidades para resolver problemas; Percepción de la atmósfera, que agrupa las variables relacionadas al clima del aula y si las actividades de enseñanza motivan a los estudiantes de desarrollar habilidad de relación interpersonal; Percepción social, que se refiere a los sistemas de apoyo disponibles para quienes sienten niveles altos de estrés.

En la mayoría de las aplicaciones realizadas a nivel internacional, como en los casos de Arabia Saudita, Australia, Gran Bretaña, Alemania y Emiratos Árabes, por ejemplo ${ }^{15-19}$ han evidenciado que el instrumento cuenta con un nivel adecuado de consistencia interna. Similares resultados se han obtenido en Latinoamérica y, específicamente, Chile $^{20}$.

Sin embargo, Jakobsson, Danielsen y Edgren ${ }^{18}$ realizaron el análisis factorial exploratorio y confirmatorio del DREEM para su muestra en Suecia y evidenciaron cinco nuevos factores asociados a su realidad educativa: (a) aprendizaje y motivación; (b) comunicación; (c) situación psicosocial; (d) organización y progresión de la enseñanza; (e) mala enseñanza, y que éstos evidenciaron un análisis de confiabilidad mayor que el modelo original. Según estos autores, ni el análisis factorial exploratorio ni confirmatorio presentaron un soporte claro para la validez de constructo propuesta por el modelo de Roff et $\mathrm{al}^{5}$. Algo similar evidenciaron De Oliveira, Edson y Schonhorst ${ }^{8}$ al hacer el análisis psicométrico del DREEM en su población.

En Chile, aunque el uso del DREEM también se ha popularizado en Medicina y los estudios locales han evaluado la confiabilidad de éste ${ }^{10,20}$, no hay estudios que aporten evidencia de su validez en población chilena, lo que guió el objetivo de la presente investigación.

\section{Metodología}

El presente estudio utiliza un diseño cuantitativo no experimental, transversal, correlacional.

\section{Participantes}

Participaron 525 alumnos universitarios de cinco escuelas de Medicina de Chile, Tabla 1. El $46,67 \%(\mathrm{n}=245)$ de los participantes eran hombres y $48 \%(n=252)$ eran mujeres, con 28 casos que no entregaron este antecedente. Sus edades se ubicaban entre los 18 y los 29 años, con una 
Tabla 1. Distribución de los estudiantes de primer año de medicina según tipo de universidad y región en la que están matriculados

\begin{tabular}{|c|c|c|c|c|}
\hline Sede Universidad & $\begin{array}{c}\text { Valparaíso } \\
\text { n (\%) }\end{array}$ & $\begin{array}{c}\text { Región } \\
\text { Metropolitana } \\
\text { n (\%) }\end{array}$ & $\begin{array}{l}\text { Bío Bío } \\
\text { n (\%) }\end{array}$ & $\begin{array}{l}\text { Total } \\
\text { n } \quad(\%)\end{array}$ \\
\hline Tradicionales* & $0 \quad(0,00)$ & $63(12,00)$ & $135(25,71)$ & $198 \quad(37,71)$ \\
\hline Privadas** & $99(18,86)$ & $133(25,33)$ & $95(18,10)$ & $327 \quad(62,10)$ \\
\hline Total & $99(18,86)$ & $196(37,33)$ & $230(43,81)$ & $525(100,00)$ \\
\hline
\end{tabular}

*Miembro del Consejo de Rectores de Universidades Chilenas, ${ }^{*}$ No miembro del CRUCH.

media de 20,45 (D.E. $=1,56) ; 51,43 \%$ provenía de colegios privados $(\mathrm{n}=270), 25,71 \%$ de subvencionados $(n=135)$ y $6,29 \%$ de municipalizados $(\mathrm{n}=33)$.

\section{Instrumentos}

Se utilizó la escala Dundee Ready Education Environment Measure (DREEM) diseñada por Roff et $\mathrm{al}^{5}$. Este instrumento está compuesto por 50 ítems que presentan factores asociados a la percepción que tiene el estudiante acerca del clima educativo. El participante debe indicar el grado en que el ítem representa una característica de él, utilizando cinco alternativas de respuesta en formato tipo Likert $(1=$ Nunca, $2=$ Pocas veces, $3=$ Algunas veces, $4=$ Habitualmente y $5=$ Siempre).

\section{Procedimiento}

Se aplicó la encuesta DREEM a estudiantes de Medicina en diferentes universidades, luego de obtener la autorización institucional de sus respectivas casas de estudio. Posteriormente, se les contactó y aplicó grupalmente el cuestionario en instalaciones de la universidad, previa realización de un consentimiento informado visado por el Comité de Ética de CONICYT.

\section{Análisis de datos}

La evaluación de las propiedades psicométricas del DREEM implicó analizar su estructura factorial mediante análisis factorial exploratorio aplicando método de extracción de ejes principales y rotación oblicua Promax. Para definir el número de factores según los criterios establecidos, se utilizó el criterio de Kaise-Guttman, MAP de Velicer y Análisis Paralelo de Horn. Para analizar la consistencia interna se aplicó coeficiente Alfa de Cronbach.

\section{Resultados}

En primer lugar se evaluó la pertinencia del análisis. Ante esto, el estadístico de adecuación muestral de Kaiser-Meyer-Olkin (KMO) fue de 0,90 y la prueba de esfericidad de Bartlett resultó estadísticamente significativa $\chi^{2}(1225)=7604,98$; $\mathrm{p}<0,001$, apoyando la adecuación del análisis factorial.

Luego se aplicaron cuatro procedimientos, complementariamente, para estimar el número de factores de la escala: 1) el criterio de Kaiser-Guttman o de raíz latente; 2) el scree test o criterio de contraste de caída (Hair et al., 2005; Martínez, Hernández y Hernández, 2006), el MAP de Velicer y el análisis paralelo de Horn (Buja y Eyuboglu, 1994).

Considerando los 50 ítems, el criterio de Kaiser-Guttman (Hair et al., 2005) identificó cuatro factores con valores propios (eigenvalues) mayores a 1,0 ; siendo éstos de 9,$41 ; 2,00 ; 1,49$ y 1,39 , los que explicarían $80,10 \%$ de la varianza total de los ítems. El scree test indicó la presencia de cuatro factores. El MAP de Velicer identificó dos factores, presentando un promedio de los cuadrados de las correlaciones entre los ítems de 0,063; encontrando el menor de los promedios de los cuadrados de las correlaciones parciales al parcializar el cuarto componente con un promedio de 0,039. Y el análisis paralelo de Horn en base a 5.000 muestras aleatorias, indicó la presencia de cuatro factores con valores propios $(9,41 ; 2,00 ; 1,49$ y 1,39$)$ sobre el percentil 95 de los valores propios obtenidos en las muestras aleatorias $(0,79 ; 0,69 ; 0,59$ y 0,54$)$.

Dado que los resultados coincidían, se procedió a evaluar la composición de la solución de cuatro factores empleando AEP y rotación oblicua Promax. Para esto se generó una matriz de confi- 
guración para cada una de estas soluciones, en la cual se identificó que 11 ítems presentaban cargas factoriales bajo 0,30 (ítems $3,13,15,26,30,31$, $32,38,40,47$ y 48 ), valor sugerido como mínimo para considerar significativa una carga factorial (Hair et al., 2005). Además, se presentaban cuatro cargas cruzadas (ítems 22, 24, 39 y 43), esto es, ítems con cargas sobre el umbral en más de un factor. A partir de este resultado se decidió eliminar el ítem que presentaba cargas más bajas (ítem 48, "La formación está centrada más en entregar conocimientos que en lograr aprendizajes"), y repetir el análisis.

Este análisis se realizó 10 veces y en cada una se fue eliminado el ítem que presentara menores cargas, hasta que se logró una solución en la que todos presentaban al menos una carga de 0,30. Luego, con los 40 ítems restantes, el coeficiente KMO arrojó 0,90 y la prueba de Bartlett fue estadísticamente significativa $\chi^{2}(780)=6078,72$; $\mathrm{p}<0,001$, apoyando la realización del análisis factorial.

Para este nuevo conjunto de ítems, el criterio de Kaiser-Guttman indicó la presencia de cuatro factores con valores propios mayores a 1,0 ; siendo éstos de 8,$10 ; 1,85 ; 1,43$ y 1,21 ; los que explicarían $89,68 \%$ de la varianza total de los ítems. En el caso del criterio de contraste de caída o scree test, el gráfico de sedimentación apuntó a la presencia de cuatro factores, Figura 1.

En tercer lugar, el MAP de Velicer identificó dos factores, presentando un promedio de los cuadrados de las correlaciones entre los ítems de
0,044; encontrando el menor de los promedios de los cuadrados de las correlaciones parciales al parcializar el cuarto componente con un promedio de 0,008 .

En tanto, el análisis paralelo de Horn en base a 5.000 muestras aleatorias, indicó la presencia de cuatro factores con valores propios $(8,10 ; 1,85$; 1,43 y 1,21) sobre el percentil 95 de los valores propios obtenidos en las muestras aleatorias $(0,64$; $0,62 ; 0,55$ y 0,49$)$.

En atención a estos resultados, se analizó la composición de la solución de cuatro factores para estos 40 ítems, encontrándose que esta vez todos los ítems presentaban cargas factoriales sobre el umbral, con tres cargas cruzadas (ítems 18, 22 y 43), Tabla 2.

A partir de la estructura factorial que se exhibe en la matriz de configuración de la Tabla 1, es posible identificar cuatro factores configurados de la siguiente manera:

- Factor I: Conformado por los ítems 21, 20, $24,16,7,2,41,44,37,45,40,14,1,22,32,43$, 17 y 3 (ordenados de mayor a menor carga), hace referencia a la calidad académica que atribuyen los alumnos al proceso en el que participan, así como a la evaluación que hacen de sus resultados de aprendizaje, ante lo cual se denominó Percepción académica. Al analizar la consistencia interna de este factor, obtuvo un coeficiente Alfa de Cronbach de $a=0,85$, con correlaciones entre los ítems y el total corregido desde $r=0,25$ (ítem 17) a $r=0,60$ (ítem 21).

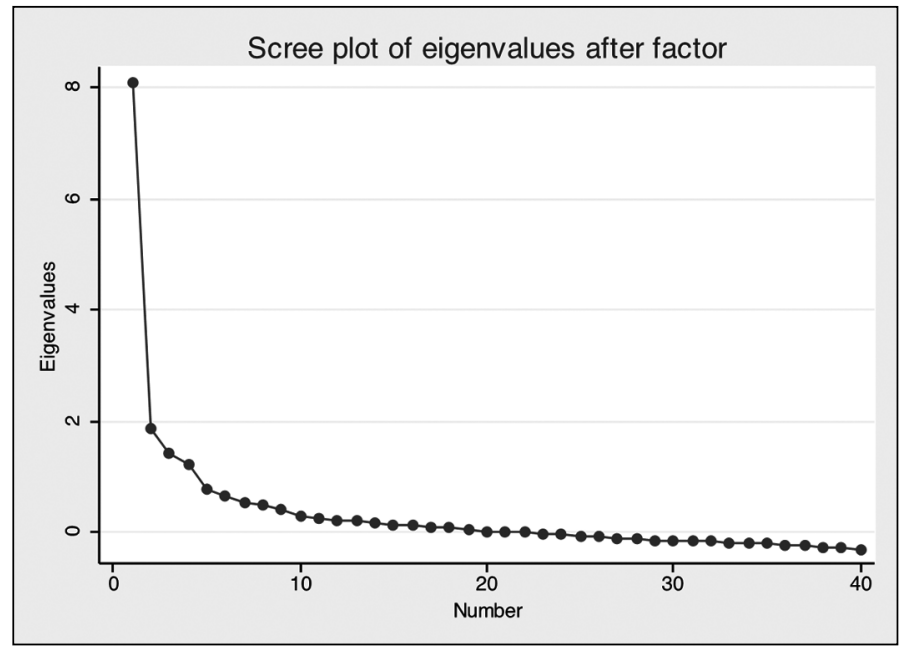

Figura 1. Gráfico de sedimentación para la Escala DREEM considerando 41 ítems (sin ítem $48,31,30,15,38,47,13,12,46$ y 26). 
Tabla 2. Matriz de configuración de la escala DREEM (40 ítems) obtenida mediante análisis de eje principal con rotación Promax

\begin{tabular}{|c|c|c|c|c|c|}
\hline ítem & Enunciado & $\mathbf{I}$ & II & III & IV \\
\hline 1 & Se me estimula a participar en las clases & $0,416^{a}$ & 0,056 & $-0,054$ & 0,001 \\
\hline 2 & Los profesores son expertos en las materias que enseñan & 0,518 & $-0,154$ & 0,071 & 0,061 \\
\hline 3 & Hay un buen sistema de apoyo para los estudiantes que sufren de estrés & 0,342 & 0,073 & 0,037 & 0,088 \\
\hline 4 & Estoy demasiado cansado para disfrutar de la carrera que estoy cursandoi & $-0,036$ & 0,557 & $-0,084$ & $-0,183$ \\
\hline 5 & Los métodos de estudio que tenía antes continúan funcionándome ahora & $-0,150$ & 0,461 & 0,053 & 0,123 \\
\hline 6 & Los profesores tienen paciencia con las personas & 0,200 & $-0,082$ & 0,305 & $-0,169$ \\
\hline 7 & A menudo, la enseñanza estimulante & 0,527 & 0,155 & $-0,087$ & $-0,041$ \\
\hline 8 & Los profesores ridiculizan a los estudiantesi & 0,092 & $-0,036$ & 0,206 & $-0,418$ \\
\hline 9 & Los profesores son autoritariosi & 0,062 & $-0,115$ & 0,140 & $-0,498$ \\
\hline 10 & Tengo confianza de que voy a aprobar mis asignaturas & $-0,128$ & 0,548 & 0,052 & $-0,116$ \\
\hline 11 & El ambiente de enseñanza es relajado en las visitas a los campos clínicos & $-0,119$ & 0,072 & 0,688 & $-0,173$ \\
\hline 14 & Rara vez me aburro en las asignaturas que estoy tomando & 0,426 & 0,156 & $-0,180$ & $-0,040$ \\
\hline 16 & La enseñanza me ayuda a desarrollar mis competencias & 0,547 & 0,057 & $-0,034$ & 0,100 \\
\hline 17 & Hacer trampa en las evaluaciones (p.e. copiar) constituye una falta grave en la carrera & 0,352 & $-0,113$ & $-0,076$ & $-0,077$ \\
\hline 18 & Los profesores tienen habilidad para comunicarse bien con las personas & 0,306 & $-0,068$ & 0,353 & $-0,115$ \\
\hline 19 & Mi vida social es buena & $-0,013$ & 0,403 & 0,231 & 0,211 \\
\hline 20 & La enseñanza está bien enfocada & 0,637 & $-0,046$ & 0,043 & 0,023 \\
\hline 21 & Siento que me están preparando bien para ejercer mi profesión & 0,726 & $-0,080$ & $-0,059$ & $-0,043$ \\
\hline 22 & La enseñanza en la carrera se preocupa de desarrollar la confianza en mí mismo & 0,412 & 0,303 & $-0,031$ & 0,041 \\
\hline 23 & El ambiente es relajado durante las clases teóricas y/o conferencias & $-0,089$ & 0,052 & 0,753 & $-0,093$ \\
\hline 24 & El tiempo destinado a las actividades de enseñanza es bien utilizado & 0,633 & $-0,183$ & 0,137 & 0,210 \\
\hline 25 & La enseñanza en la carrera pone demasiado énfasis en el aprendizaje de detallesi & $-0,168$ & 0,069 & $-0,036$ & $-0,331$ \\
\hline 27 & Soy capaz de memorizar todo lo que me es necesario & 0,049 & 0,505 & 0,079 & 0,202 \\
\hline 28 & Rara vez me siento solo & $-0,054$ & 0,498 & 0,140 & 0,252 \\
\hline 29 & Los profesores nos dan información adecuada sobre cómo va nuestro desempeño en los estudios & 0,267 & 0,050 & 0,233 & 0,319 \\
\hline 32 & En la carrera, los profesores nos hacen críticas constructivas & 0,364 & $-0,024$ & 0,135 & 0,084 \\
\hline 33 & Me siento cómodo, socialmente, en clases & 0,087 & 0,253 & 0,374 & 0,149 \\
\hline 34 & El ambiente en los seminarios y clases es relajado & $-0,116$ & 0,073 & 0,787 & $-0,071$ \\
\hline 35 & Mi experiencia en la carrera ha sido desalentadorai & $-0,072$ & $-0,556$ & 0,005 & 0,221 \\
\hline 36 & Soy capaz de concentrarme bien & $-0,021$ & 0,582 & 0,053 & $-0,006$ \\
\hline 37 & Los profesores dan ejemplos claros & 0,477 & $-0,093$ & 0,160 & $-0,046$ \\
\hline 39 & Los profesores se molestan o se alteran en las clases ${ }^{i}$ & $-0,047$ & $-0,084$ & 0,243 & $-0,573$ \\
\hline 40 & Los profesores van bien preparados a sus clases & 0,429 & $-0,167$ & 0,210 & $-0,035$ \\
\hline 41 & Estudiar esta carrera me está ayudando a desarrollar mi capacidad para resolver problemas & 0,503 & 0,127 & $-0,025$ & 0,057 \\
\hline 42 & El grado en que disfruto mis estudios pesa más que la tensión que éstos me generan & 0,145 & 0,534 & $-0,126$ & $-0,111$ \\
\hline 43 & El ambiente de la carrera me motiva a aprender & 0,355 & 0,341 & 0,065 & $-0,123$ \\
\hline 44 & La manera de enseñar en la carrera me estimula a aprender por mí mismo & 0,494 & 0,175 & $-0,090$ & 0,037 \\
\hline 45 & Mucho de lo que tengo que aprender me parece relevante para mi carrera como médico & 0,438 & 0,025 & $-0,105$ & $-0,260$ \\
\hline 49 & En la carrera siento que puedo hacer todas las preguntas que quiera & 0,197 & 0,091 & 0,339 & $-0,082$ \\
\hline 50 & Los estudiantes causamos molestia para los profesoresi & 0,118 & 0,078 & 0,258 & $-0,348$ \\
\hline
\end{tabular}

aLas cursivas indican cargas superiores a 0,30. ítems inversos. 
- Factor II: Incluye los ítems 36, 10, 42, 27, 28, 5, 19,35 y 4 , que hacen referencia a las emociones positivas que los estudiantes experimentan durante la carrera, tales como confianza, agrado y energía, por lo que se denominó Experiencia académica. Obtuvo un $a=0,76$ y correlaciones entre los ítems y el total corregido desde $r=0,33$ (ítem 5) a $r=0,53$ (ítem 36).

- Factor III: Incluye los ítems 34, 23, 11, 18, 49, 33 y 6 , que hacen referencia a la comodidad y tranquilidad que los estudiantes experimentan durante las actividades académicas, por lo que se denominó Percepción de la atmósfera, y su confiabilidad fue de $a=0,79$, con correlaciones entre los ítems y el total corregido desde $r=0,38$ (ítem 6) a $r=0,68$ (ítem 34).

- Factor IV: Incluye los ítems 39, 9, 8, 50, 25 y 29, que aluden a la percepción que se tiene de los profesores y de su interés y disposición hacia los alumnos, por lo que se denominó Percepción de los docentes y presentó una consistencia interna de $a=0,50$, con correlaciones entre los ítems y el total corregido desde $r=-0,06$ (ítem 29) a $r=0,45$ (ítem 8).

\section{Discusión}

A partir de los resultados obtenidos por este estudio, se encontró que el instrumento DREEM presenta una estructura factorial diferente entre los estudiantes de Medicina chilenos que el propuesto por Roff ${ }^{5}$, lo cual presenta implicaciones relevantes para el uso posterior del instrumento en esta población, sobre todo considerando que ya hay otros estudios que al evaluar la estructura factorial han diferido de la propuesta original del autor $^{18}$.

La mayoría de los estudios realizados hasta el momento, tanto en el mundo ${ }^{5,8}$ como en Chile ${ }^{20,10}$, se han enfocado en hacer un análisis de la consistencia interna, considerando los 50 ítems del diseño original entorno a los factores propuestos por Roff 6 . Sin embargo, la confiabilidad hace referencia al grado de precisión de la medición y no a si la interpretación que hacemos de ésta es adecuada, es decir, su validez ${ }^{21}$.

En este punto, la inquietud que se plantea es si en Chile es adecuado obtener e interpretar las cinco puntuaciones del DREEM que proponen Roff et al. ${ }^{5}$; el presente estudio muestra que, al menos en nuestro país, no hay evidencia que apoye esta propuesta. Por el contrario, el instrumento estaría midiendo cuatro dimensiones, las cuales siguen mostrando coherencia teórica para el estudio del ambiente educativo y una relevancia práctica si se quiere utilizar como herramienta de diagnóstico de la realidad de las escuelas de Medicina. Así, la propuesta identificada permite diferenciar la opinión de los estudiantes sobre los procesos formativos en los que participan, aludiendo a una dimensión más académica del ambiente; sobre los afectos que este genera, haciendo referencia a un aspecto más subjetivo; sobre su percepción de los aspectos no académicos del entorno, relevando su interacción con éste, y sobre los académicos, que constituyen el principal agente con el que interactúan en el proceso.

Adicionalmente, este estudio mostró que 10 ítems del DREEM no presentaban una relación suficiente con los factores identificados como para justificar su inclusión en el instrumento. Los 10 ítems eliminados corresponden a áreas como las relaciones interpersonales, la percepción del ambiente de estudio en la institución educativa, los espacios y la gestión del aprendizaje, todos aspectos ya contenidos en la estructura de cuatro factores. Por tanto, su exclusión es atribuible a problemas de formato de los ítems. Esto es fundamental, ya que la interpretación que se puede recoger de los resultados influye en la toma de decisiones que se realizan en diversos contextos educativos. Muchos elementos que se podrían considerar en programas de mejora educativa se basan en los resultados diagnósticos obtenidos por instrumentos que no resguardan todos los criterios de validez y de confiabilidad.

El presente estudio, por tanto, muestra una estructura factorial de cuatro factores para cuarenta ítems, que cuenta con evidencia empírica para ser usada en la realidad chilena. Pese a lo anterior, es necesario continuar con otros estudios de la estructura factorial del DREEM que incluyan un análisis factorial confirmatorio de las diferentes estructuras propuestas y abarquen una mayor heterogeneidad geográfica e institucional de las escuelas de Medicina. Así también, se considera necesario recabar otras evidencias de validez del instrumento, como su validez convergente, discriminante y de criterio en nuestra realidad nacional y latinoamericana. 
Agradecimientos: a Olga Matus, académica del Departamento de Educación Médica de la Facultad de Medicina Universidad de Concepción, por su apoyo en el proceso de recolección de datos y a todos los estudiantes que participaron en esta investigación.

\section{Referencias}

1. OCDE. Revisión de Políticas Nacionales de Educación: La Educación Superior en Chile. Chile: Organización para el Desarrollo y la Cooperación Económicas y el BIRD/Banco Mundial; 2009.

2. Hernández A. Los desafíos de la docencia universitaria. Revista Educación de la Universidad de Costa Rica 2002; 26 (2): 117-24.

3. Polanco Y. La unidad docencia-investigación. Universidad de Carabobo, Facultad de Ciencia de la Educación. Rev Educ Cienc Salud 2002; 20: 107-15.

4. Gros B. Tendencias actuales de la investigación en docencia universitaria. Universidad de Barcelona. Revista de educación superior en Farmacia 2007; 1: 2-12.

5. Roff S, McAleer S, Harden R, Al-Qahtani M, Uddin A, Deza H, et al. Development and Validation of the Dundee Ready Education Environment Measure (DREEM). Med Teach 1997; 19 (4): 295-9.

6. Roff S. The Dundee Ready Education Environment Measure (DREEM)-a generic instrument for measuring students' perceptions of undergraduated health professions curricula. Med Teach 2005; 27 (4): 322-5.

7. Till H. Climate studies: can students perceptions of the ideal educational environment be of use for institutional planning and resource utilization? Medical Teacher 2005; 27 (4): 332-7.

8. De Oliveira G, Edson J, Schonhorst L. Psychometric of the Dundee Ready Education Environment Measure (DREEM) applied to medical residents. Med Teach 2005; 27 (4): 343-7.

9. Murillo F. Investigación Iberoamericana. Sobre Eficacia Escolar. Chile: Edición Andrés Bello; 2007.

10. Díaz-Véliz G, Mora S, Bianchi R, Gargiulo P, Terán C, Gorena D, et al. Percepción de los estudiantes de medicina del ambiente educativo en una facultad con currículo tradicional (UCH-Chile) y otra con currículo basado en problemas (UNC-Argentina). Educ Med 2011; 14 (1): 27-34.

11. Pinparyon P, Roff S, McAleer S, Poonchai B, Pemba S. Educational environment, student approaches to learning and academic achievement in a Thai nursing school. Med Tech 2000; 22: 355-64.

12. Ortiz L. El legado flexneriano en el siglo 21. Rev Educ Cienc Salud 2010; 7 (1): 36-8.

13. Dolmans D, Wolfhagen I, Van Merriënboer J. Twelve tips for implementing whole-task curricula: How to make it work. Med Teach 2013; 15: 1-5.

14. Hutchins E.The1960s medical school graduate: his perceptions of this faculty, peers, and environment. Journal of Medical Education 1961; 36: 322-9.

15. Bassaw B, Roff S, Mcaleer S, Roopnarinesingh S, De Lisle J, Teelucksingh S, Gopaul S. Students' perspectives on the educational environment. Med Teach 2003; 25 (5): 522-6.

16. Al-Hazimi A, Al-Hyiani A, Roff S. Perceptions of the educational environment of the medical school in King Abdul Aziz University. Med Teach 2004; 26 (6): 570-3.

17. Carmody D, Jacques A, Denz-penhey H, Puddey, Newnham J. Perceptions by medical students of their educational environment for obstetrics and gynaecology in metropolitan and rural teaching sites. Med Teach 2009; 31: 596-602.

18. Jakobsson U, Danielsen N, Edgren G. Psychometric evaluation of the Dundee Ready Educational Environment Measure: Swedish version. Medical Teacher 2011; 33: 267-74.

19. Rotthoff T, Ostapczuk M, De Bruin J, Decking U, Schneider M, Ritz-timme S. Assesing the learning environment of a faculty: Psychometric validation of the German version of the Dundee Ready Education Environment Measure with students and teachers. Med Teach 2011; 33: 624-36.

20. Herrera C, Pacheco J, Rosso F, Cisterna C, Aichele D, Becker S, et al. Evaluación del ambiente educacional pre-clínico en seis Escuelas de Medicina en Chile. Rev Med Chile 2010; 138: 677-84.

21. Martínez R, Hernández M, Hernández M. Psicometría. Madrid: Alianza; 2006. 